\title{
Ajuste de las especificaciones técnicas del test de viabilidad por tetrazolio en semillas de Cenchrus ciliaris L. (buffel grass)
}

Agüero, C. G. y Rolando, R. O.

\begin{abstract}
RESUMEN
El objetivo del estudio fue establecer lineamientos para realizar el test de viabilidad de semillas por tetrazolio en Cenchrus ciliaris utilizando los protocolos de Panicum (ISTA, 2012). Se trabajó con fascículos (unidad de dispersión) de Cenchrus ciliaris del cultivar Texas de los cuales se extrajeron cariópsides para realizar los ensayos. Se determinó el método apropiado de corte de cariópsides y los tiempos de tinción; se probaron dos concentraciones de solución de tetrazolio. Además, se desarrollaron protocolos de evaluación. Las condiciones adecuadas para realizar el test de viabilidad son: $18 \mathrm{~h}$ de humedecimiento entre papel a temperatura de $20^{\circ} \mathrm{C}$; corte longitudinal incompleto a través del embrión; concentración de tetrazolio al 0,5 o 1 \% y 10 h de tinción a $30^{\circ} \mathrm{C}$. Los mapas de tinción permiten diferenciar de forma precisa la viabilidad de las cariópsides.
\end{abstract}

Palabras clave: Cenchrus ciliaris; viabilidad; tetrazolio; cariópsides; protocolos.

Agüero, C. G. and Rolando, R. O., 2019. Adjustment of the technical specifications for the Tetrazolium viability test in Cenchrus ciliaris $L$. (buffel grass) seeds. Agriscientia 36 (2): 81-88

\section{SUMMARY}

The objective of the study was to establish guidelines for the viability test of seeds by tetrazolium in Cenchrus ciliaris using the Panicum protocols (ISTA, 2012). We worked with fascicles (dispersion unit) of Cenchrus ciliaris of the Texas cultivar from which caryopses were extracted to perform the tests. The appropriate method for cutting caryopses and the staining times were determined and two concentrations of tetrazolium solution were tested. In addition, after adjusting the conditions, evaluation protocols were developed. The adequate conditions to perform the viability test are $18 \mathrm{~h}$ of seed wetting between papers at a temperature of $20^{\circ} \mathrm{C}$, incomplete longitudinal cut through 
the embryo, tetrazolium concentration at 0.5 or $1 \%$ and 10 hours of staining at $30{ }^{\circ} \mathrm{C}$. The staining maps allow differentiating accurately the viability of the caryopses.

Key words: Cenchrus ciliaris; viability; tetrazolium; caryopses; protocols.

Agüero, C. G. y Rolando, R. O.: Facultad de Ciencias Agropecuarias, Universidad Nacional de Córdoba (FCA-UNC). Laboratorio de Análisis de Semillas, Investigación, Docencia y Servicios (LASIDYS). Félix Marrone 746, Ciudad Universitaria, 5000, Córdoba, Argentina. Correspondencia a: aguerocesar@agro.unc.edu.ar

\section{INTRODUCCIÓN}

La ganadería constituye uno de los principales sistemas de producción agropecuaria y en Argentina el proceso de expansión de la agricultura desplazó la frontera ganadera a sitios marginales para dar lugar a la producción de soja (Reartes, 2003). Estudios demostraron que las gramíneas megatérmicas perennes como buffel grass (Cenchrus ciliaris L.), gatton panic (Megathyrsus maximus (Jacq.) B.K. Simon \& S.W.L. Jacobs ex Panicum maximum Jacq.) y grama rhodes (Chloris gayana Kunth) pueden duplicar o triplicar la cantidad de forraje producido respecto a pastizales naturales. Para garantizar una buena implantación resulta crucial obtener simientes de calidad (Ayerza, 1981; Pérez, 2005).

La calidad en las semillas se define por atributos físicos, fisiológicos, genéticos y sanitarios (Peske, Rosenthal y Madeiros Rota, 2003). Específicamente, el atributo fisiológico que en las semillas está dado por la capacidad para desempeñar funciones vitales es determinado en laboratorio mediante los ensayos de germinación, viabilidad y vigor. El test de viabilidad por tetrazolio (TZ) descripto por AOSA (Association of Official Seed Analysts, 2009) y por ISTA (International Seed Testing Association, 2012) permite a través de la actividad de las enzimas deshidrogenasas (Smith y Throneberry, 1951; Smith, 1952; Copeland, Bruce y Midyette, 1959; Bulat, 1961; Moore, 1973; AOSA, 1983; Krzyzanowski, Vieira, Franca Neto, 1999) conocer el número de semillas viables y no viables que un lote de semillas posee (Ellis, ،Hong y Roberts, 1985).

Un inconveniente en la determinación de la viabilidad en semillas de $C$. ciliaris es la obtención de material para los ensayos. En especies como Chloris gayanay Panicumspp. las reglas establecen que espiguillas sin cariópside (semillas) deben considerarse no viables. En Cenchrus, género no incluido en el capítulo de viabilidad de ISTA (2012) pero sí en las Hojas de Trabajo de Tetrazolio (ISTA, 2003), no especifican cómo expresar a los fascículos vacíos (unidad de dispersión) cuando se realiza viabilidad por tetrazolio. Al respecto, Agüero, Pereyra y Rolando (2017) mencionaron que la evaluación de pureza descripta en ISTA necesita, para una valoración precisa y útil, determinar el porcentaje de fascículos llenos, o sea que contengan cariópside/s en su interior.

En diversas especies las reglas ISTA (2012) sugieren previo a la tinción realizar cortes en las semillas de manera de permitir la exposición de los tejidos a la solución de tetrazolio y facilitar la evaluación. Para cariópsides pequeños de géneros como Brachiaria spp., Chloris gayana, Dactylis spp.y Panicum spp. se recomiendan cortes como el longitudinal incompleto a través del embrión y transversal completo. Dentro del género Cenchrus, ISTA (2003) prescribe cortes longitudinales a través de la mitad distal del endospermo.

Por otra parte, una particularidad del test de viabilidad es que puede diferenciar las semillas muertas de aquellas que se encuentran en dormición (Gosling, 2003). Según Sharif-Zadeh y Murdoch (2000), la dormición en buffel grass es una de las razones principales de la baja germinación de esta especie. Tanto el ensayo de germinación como el de viabilidad suelen presentar inconvenientes al analizar semillas de C. ciliaris. La germinación en pocas ocasiones alcanza valores satisfactorios, incluso aplicando los tiempos de ensayo y pretratamientos de ruptura de dormición indicados por ISTA (Agüero et al., 2017); mientras que la metodología para determinar la viabilidad por TZ se encuentra definida de forma general en ISTA (2003). Por lo cual, los tipos de daños expresados en los mapas topográficos, la metodología propuesta y la ausencia de la definición de cómo 
considerar los fascículos sin cariópsides pueden provocar discrepancias en la evaluación de la viabilidad. Entonces, dado que el protocolo de Panicum en ISTA (2012) guarda similitudes con los establecidos por las Hojas de Trabajo para el Test de Viabilidad (ISTA, 2003) para C. ciliaris y considerando que éstas destacan la necesidad de profundizar los conocimientos en la realización de los ensayos de viabilidad, el objetivo del trabajo fue ajustar las especificaciones técnicas del test de viabilidad de tetrazolio aplicados a semillas de C. ciliaris.

\section{MATERIALES Y MÉTODOS}

Se estudiaron fascículos de C. ciliaris cultivar Texas ingresados durante julio de 2013 al Laboratorio de Análisis de Semillas, Investigación, Docencia y Servicios (LASIDYS), FCA-UNC. Las cariópsides se extrajeron de los fascículos según la metodología de Agüero et al. (2017). El acondicionamiento de las cariópsides se efectuó de acuerdo a pautas fijadas por ISTA (2012) para Panicum spp. Se realizaron los tratamientos descriptos a continuación. Cada tratamiento estuvo formado por cuatro repeticiones de 25 cariópsides. Cada pool de cariópsides de cada repetición se colocó entre papel humedecido con agua destilada en cámara a $20{ }^{\circ} \mathrm{C}$ durante un período de $18 \mathrm{~h}$ y dentro de cajas de Petri para evitar la deshidratación del material. La observación de cariópsides se realizó con lupa Motic SMZ-168 Serie trilocular (50x) equipada con conjunto para video-microscopía digital 1.3 MP MotiCAM.

\section{Evaluación de tipos de corte}

Para la realización de la prueba se siguieron las especificaciones de Panicum spp. (ISTA, 2012). Según lo prescripto, la temperatura de tinción fue de $30{ }^{\circ} \mathrm{C}$, la concentración de la solución de tetrazolio del $1 \%$ y el tiempo de tinción 18 h. Se evaluó en cada tratamiento el porcentaje de cariópsides teñidos. Los tipos de corte realizados fueron los siguientes:

A- Testigo: cariópsides intactas sin cortar.

B- Corte longitudinal incompleto: corte con bisturí por la región media del embrión y hasta 3/4 del endospermo.

C- Corte longitudinal completo: corte total con bisturí por la región media del embrión y endospermo. Luego del corte se eliminó una de las mitades.

D- Corte transversal: corte con bisturí encima de la región donde se ubica el embrión descartando el resto superior de la cariópside.

\section{Tiempo óptimo de tinción y concentración de tetrazolio}

Conforme a los resultados del punto anterior, se utilizó el método de corte longitudinal incompleto (ver resultados). La tinción se llevó a cabo en estufa a temperatura de $30{ }^{\circ} \mathrm{C}$ en oscuridad. Las concentraciones de tetrazolio fueron de 0,5 y $1 \%$. El progreso de la tinción se evaluó cada 2 h retirando de la solución de tetrazolio aquellas cariópsides con sus estructuras esenciales teñidas. Se evaluó el porcentaje de cariópsides teñidos. Los ensayos
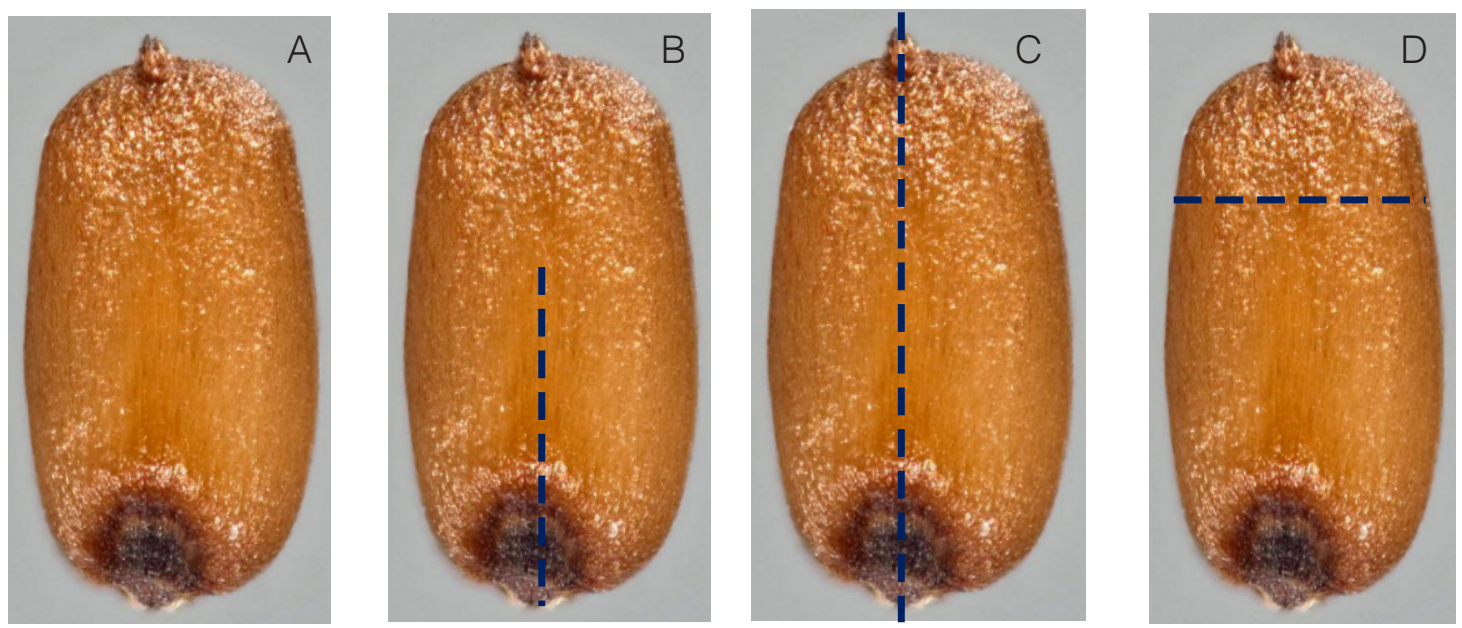

Figura 1. Tratamientos de cortes realizados en las cariópsides de C. ciliaris Texas. El sitio de corte se muestra con línea punteada: A) testigo (sin cortar), B) corte longitudinal incompleto, C) corte longitudinal completo, D) corte transversal completo. 
se extendieron hasta que no se observó tinción de nuevas cariópsides.

\section{Determinación de los patrones de tinción}

Se utilizó la técnica de corte, concentración de tetrazolio y tiempos que resultaron de ensayos previamente realizados (corte longitudinal incompleto; concentración TZ al 0,5\%; tiempo de tinción 10 h). Las cariópsides se fotografiaron con un equipo de microscopía óptica Confocal Olimpus LEXS OLS 4000. Se usaron de referencia protocolos de viabilidad de semillas de Poáceas pequeñas obtenidos en trabajos realizados en la Estación Experimental INTA Oliveros, de protocolos ISTA (2012) para Poáceas (Avena, Hordeum, Secale y Triticum) y de las Hojas de Trabajo de Tetrazolio para Cenchrus (ISTA, 2003).

\section{Diseño experimental y análisis estadístico}

El análisis estadístico se realizó empleando el software InfoStat (Di Rienzo et al., 2015). Para detectar diferencias se utilizó el análisis de la varianza. Para evaluar los tipos de corte se observó la respuesta de tinción al aplicar los tipos de cortes. Los ensayos de tiempo de tinción y concentración de TZ incluyeron en el modelo el factor tiempo y el factor concentración de TZ y la interacción entre ambos para un diseño completamente aleatorizado. En todos los análisis las comparaciones entre las medias de tratamientos se realizaron con la prueba LSD de Fisher. El nivel de significación se estableció en 0,05.

\section{RESULTADOS Y DISCUSIÓN}

\section{Tipo de corte en cariópsides}

El test de comparación de medias manifestó que no hubo diferencias significativas $(p<0,05)$ entre tratamientos de corte y que el porcentaje de semillas teñidas del testigo fue menor (Figura 2). Las cariópsides intactas presentaron un $33 \%$ de semillas sin teñir, mientras que con el corte longitudinal incompleto se obtuvo el mínimo valor de cariópsides no teñidas (8\%).

Se observó que los tratamientos de corte fueron más apropiados por sobre el testigo, el cuál necesitó de tiempos mayores de exposición

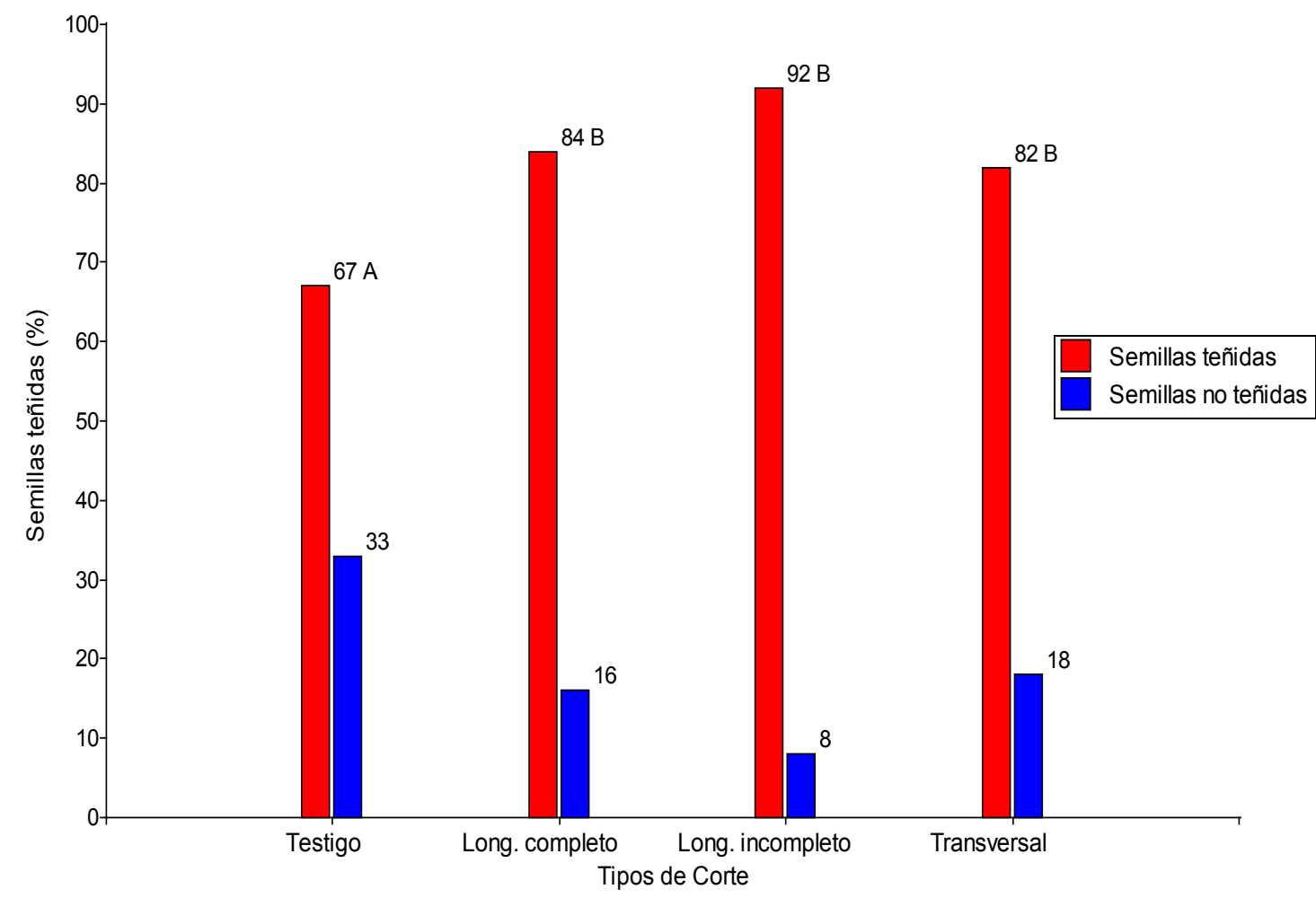

Figura 2. Semillas teñidas y no teñidas (\%) de C. ciliaris al comparar distintos tratamientos de cortes de cariópsides. Letras iguales indica que no existieron diferencias significativas entre tratamientos (Fisher $\mathrm{p}<0,05$ ). 
a la solución de tetrazolio para teñir de forma similar a los cortados. De acuerdo a Moore (1987), para analizar correctamente, es imprescindible visualizar el aspecto interno de los tejidos; tales características difícilmente sean observadas en semillas intactas o sin cortes previos en la región embrional. La falta de tinción del testigo no fue consecuencia de la mortalidad de células, sino de la escasa difusión de la solución de TZ al interior de las cariópsides. Por lo tanto, utilizar cariópsides sin cortar implica un mayor tiempo de incubación en estufa y la necesidad de realizar cortes adicionales para una mejor evaluación.

Debido a que no fueron halladas diferencias entre tratamientos, el tipo de corte a recomendar depende de la factibilidad técnica. Por consiguiente, el corte transversal presenta la desventaja de requerir cortes adicionales para poder visualizar las estructuras internas de las semillas, como el caso de las cariópsides sin cortar. El corte longitudinal completo descarta una mitad de la cariópside con lo cual hay posibilidad de subestimar o sobrestimar la viabilidad real del material. De esta forma, el corte longitudinal incompleto es el más adecuado, por ser el más simple de ejecutar, no requerir cortes adicionales y favorecer la tinción. La posición centrada del embrión en Cenchrus no es un impedimento para la realización de las incisiones, más bien las beneficia, aunque el tamaño reducido de las cariópsides requirió el uso de lupa binocular y de elementos con adecuado filo.

Tanto en las reglas ISTA (2012) para Panicum como en ISTA (2003) para Cenchrus los cortes sugeridos no son a través de la zona embrionaria, por lo cual recomiendan la disección de las cariópsides luego de la tinción. En los ensayos los cortes que requirieron de cortes posteriores (testigo y transversal completo) fueron los más dificultosos de efectuar a causa del humedecimiento excesivo de los tejidos seminales.

\section{Determinación del tiempo de tinción y concentración de tetrazolio}

El test de medias reveló diferencias significativas $(p<0,05)$ en el porcentaje de semillas teñidas entre las distintas concentraciones de TZ y entre los tiempos de tinción. Observándose interacción entre los factores tiempo y concentración de TZ $(p=0,0334)$. La Figura 3 muestra los porcentajes de

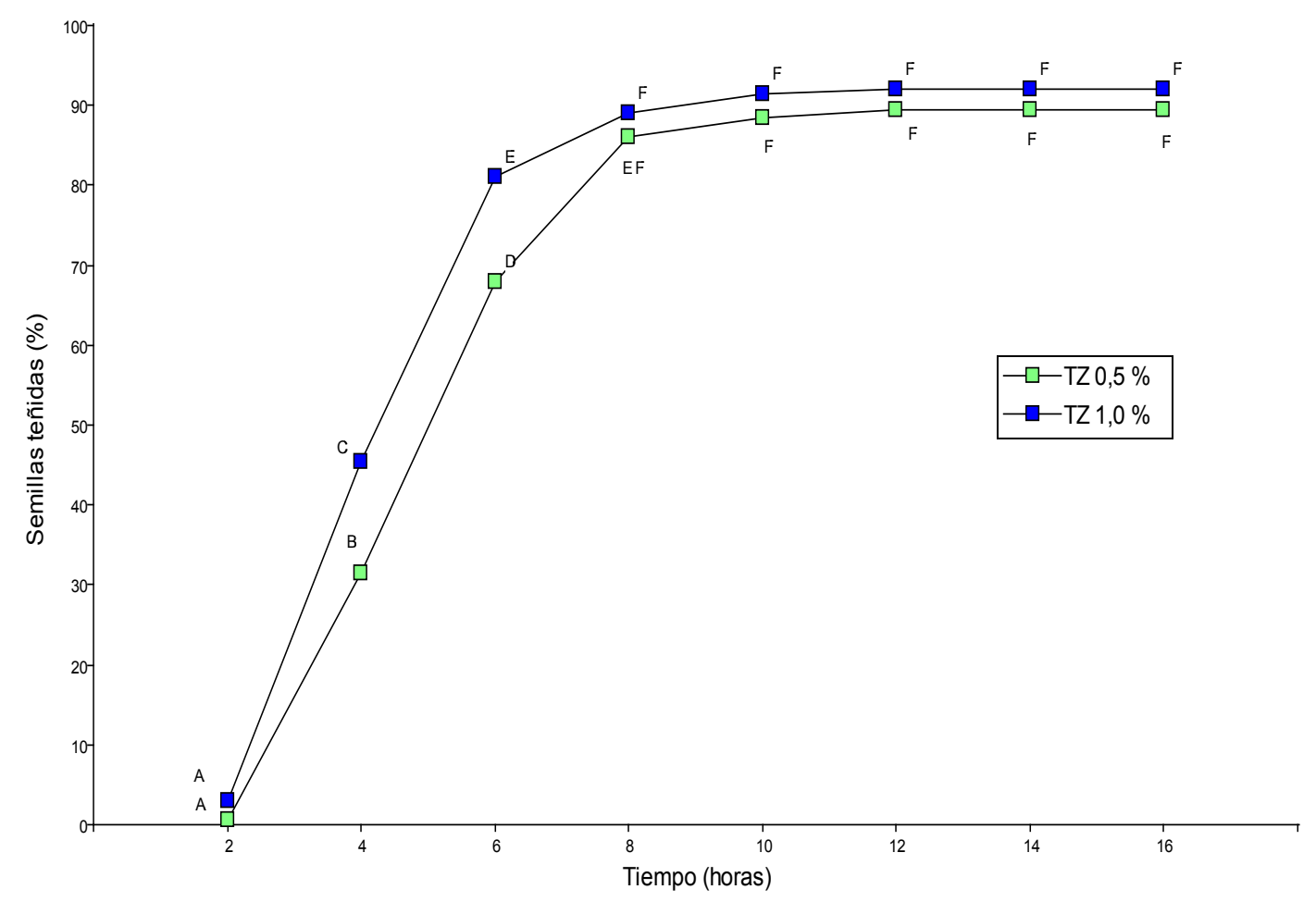

Figura 3. Porcentaje de tinción de semillas de C. ciliaris sometidas a diferentes concentraciones de tetrazolio y tiempos de tinción. Letras iguales indican que no hubo diferencias significativas entre las concentraciones de TZ en los distintos tiempos de tinción (Fisher $\mathrm{p}<0,05$ ). 
semillas teñidas de las diferentes concentraciones de TZ $(0,5$ y $1 \%)$ en cada momento de evaluación $(2,4,6,8,10,12,14$ y $16 \mathrm{~h})$.

Para las dos concentraciones de TZ, el porcentaje de semillas teñidas aumentó hasta las diez horas de iniciado el ensayo. A las $2 \mathrm{~h}$, no se encontraron diferencias entre medias, siendo el porcentaje de semillas teñidas escaso para las dos concentraciones. Durante las 4 y $8 \mathrm{~h}$ se registraron diferencias obteniéndose, con la concentración de TZ al $1 \%$, los mayores porcentajes de tinción. En lecturas posteriores a las $8 \mathrm{~h}$ las diferencias no resultaron significativas.

Los resultados revelan que en $C$. ciliaris un test de viabilidad puede realizarse en aproximadamente 28 h: 18 h de prehumedecimiento y 10 h de tinción. Estos tiempos son menores a los recomendados por ISTA (2003) para Cenchrus que prescriben 18 h de tinción, aunque con cortes longitudinales a través de la mitad distal del endospermo, lo que puede retardar la difusión del tetrazolio hacia el embrión. La utilización de solución de TZ al 0,5 \% como al 1 \% resultó indistinta.

El tiempo de evaluación dependerá del entrenamiento del analista. Estos datos son muy importantes en la especie estudiada, la cual tiene un prolongado tiempo de ejecución del análisis de germinación y cuya dormición puede inclusive enmascarar expresión de la germinación (Agüero et al., 2017). Conclusiones similares fueron expresadas por Delouche, Still, Raspet y Lienhard (1962) para semillas de Poáceas, entre ellas Cynodon dactylon (L.) Pers. y Festuca arundinacea Schreb.

\section{Patrones de tinción}

Las semillas viables sin defectos y las no viables (sin teñir) fueron las más frecuentes. Las estructuras consideradas esenciales se encuentran identificadas en una cariópside viable sin defectos (Figura 4). Sin embargo, para facilitar la evaluación y a causa de la diversidad de daños posibles, se diferenciaron categorías de tinción. Dichas categorías se agruparon en: cariópsides viables completamente teñidas (Figura 4); viables con defectos (Figuras $5 \mathrm{~A}, \mathrm{~B}$ y $\mathrm{C}$ ); no viables con defectos (Figuras 6 A, B, C, D) y cariópsides sin tinción (Figura 6 E).

Los patrones de coloración observados son similares a los expuestos en las reglas ISTA (2003), aunque éstos no expresan la totalidad de daños posibles de ser hallados, probablemente por el exiguo tamaño de las semillas. Los daños fueron semejantes a los plasmados en los protocolos

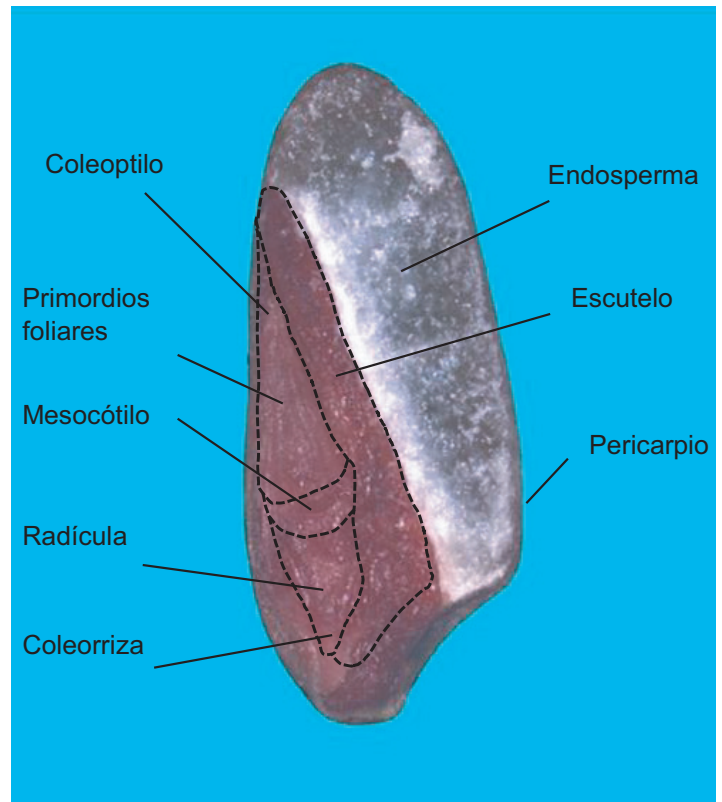

Figura 4. Cariópside viable de C. ciliaris completamente teñido con tetrazolio. Con línea de puntos se destacan áreas esenciales para la evaluación de viabilidad.

ISTA (2012) para cariópsides de mayor tamaño como de Avena, Hordeum, Secale y Triticum. La minuciosidad de la evaluación dependerá por lo tanto, del uso de lupa binocular con adecuada iluminación.

\section{CONCLUSIONES}

Se concluye que los protocolos de Panicum (ISTA) no son aplicables para Cenchrus ciliaris por dos motivos: en $C$. ciliaris los tiempos de tinción son de $10 \mathrm{~h}$ y el método de corte adecuado es el longitudinal incompleto a través del embrión y hasta 3/4 del endospermo. De esta manera, a partir de los resultados obtenidos en este estudio, se propone el siguiente protocolo para determinar la viabilidad por tetrazolio en cariópsides de $C$. ciliaris: 1) realizar cortes longitudinales incompletos, los cuales permiten determinar más certeramente la viabilidad, ya que favorece la tinción de los tejidos y la visualización de las estructuras a evaluar; 2) para la preparación y tinción de cariópsides de C. ciliaris: 2 a) realizar el humedecimiento de las semillas entre papel durante $18 \mathrm{~h}$ a $20{ }^{\circ} \mathrm{C}$; $2 \mathrm{~b}$ ) utilizar la concentración de tetrazolio al 0,5 o al 1 $\%$ y $10 \mathrm{~h}$ de incubación a $30^{\circ} \mathrm{C}$. Los patrones de tinción realizados permiten diferenciar de forma exacta la viabilidad de manera semejante a otras semillas de Poáceas. 

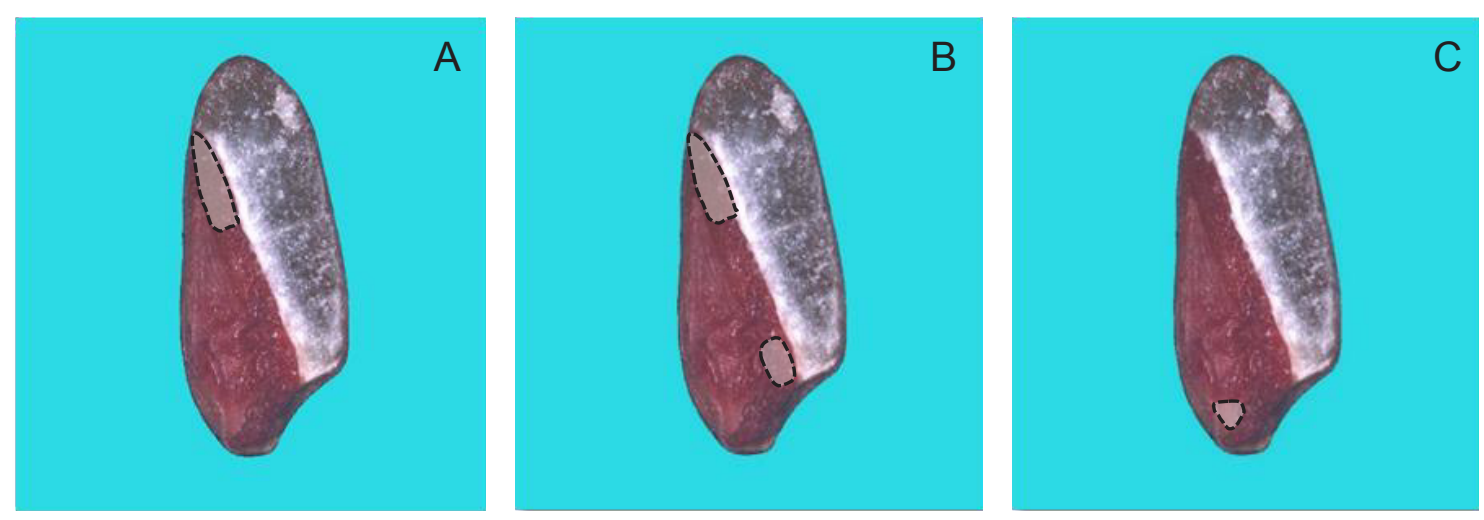

Figura 5. Cariópsides viables de C. ciliaris con defectos, luego de la tinción con tetrazolio: A) escutelo con extremo superior menor o igual a 1/3 sin teñir o necrosado; B) escutelo con extremos superior e inferior menor o igual a 1/3 sin teñir o necrosado; C) coleorriza sin teñir o necrosada.
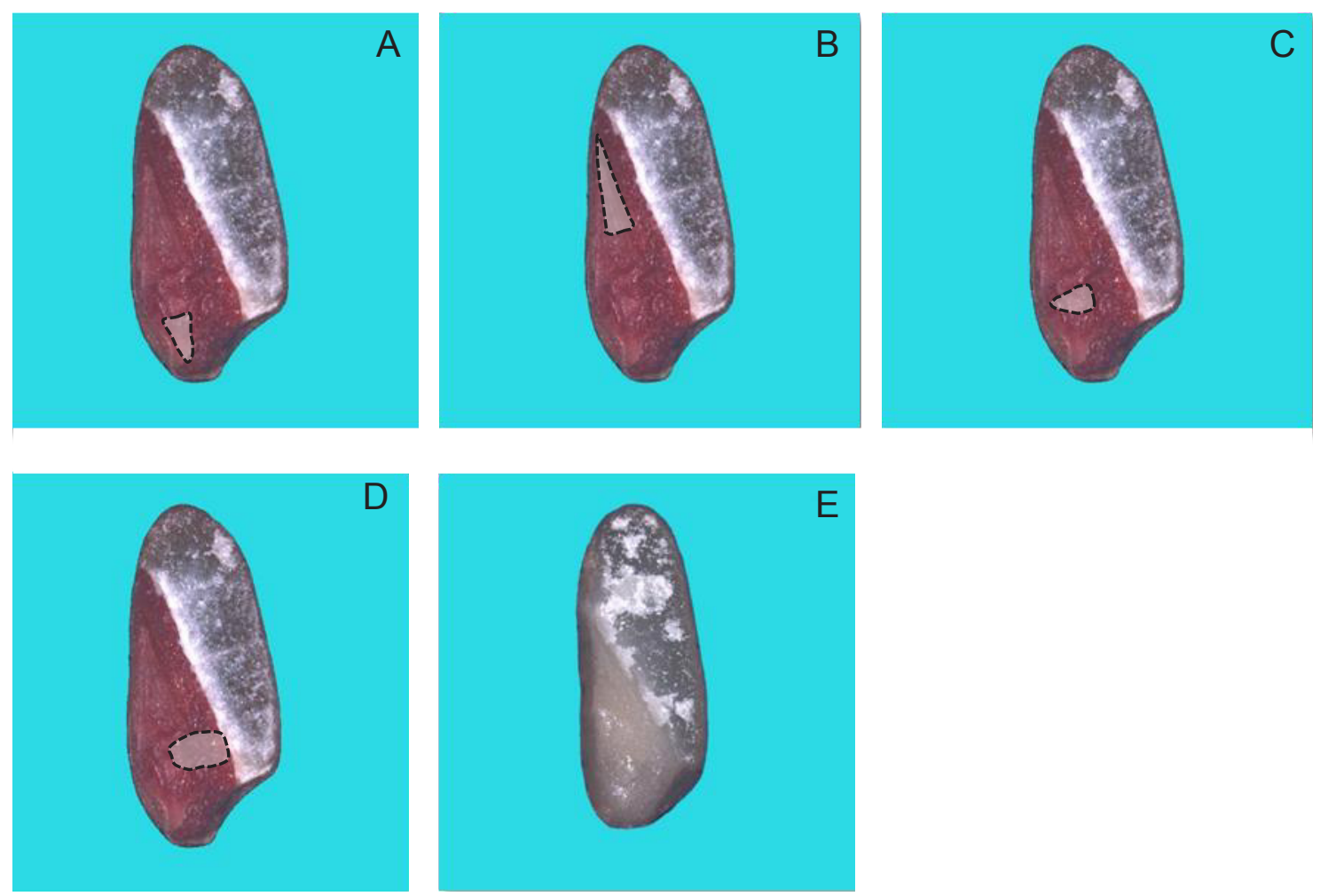

Figura 6. Cariópsides no viables de C. ciliaris luego de la tinción con tetrazolio: A) coleorriza y radícula sin teñir o necrosada; B) extremo coleoptilo sin teñir o necrosado; C) mesocótilo sin teñir o necrosado; D) escutelo banda unión con mesocótilo sin teñir o necrosado; E) cariópside no viable, sin tinción.

\section{AGRADECIMIENTOS}

A la Secretaría de Ciencia y Técnica UNC por el subsidio otorgado.

A los ingenieros agrónomos Margot Tablada, Jacqueline Joseau y Cristiano Casini por sus valiosos aportes.

\section{BIBLIOGRAFÍA}

Agüero, C. G., Pereyra, G. y Rolando, R. O. (2017). Método alternativo de germinación para determinar la calidad de semillas en Buffel Grass (Cenchrus ciliaris L.). Agriscientia, 34 (1) 47-58. doi: 10.31047/1668.298x. v34.n1.17465 
Association of Official Seed Analysts (AOSA) (2009). Seed Vigor Testing Handbook. Ithaca, NY, USA: Association of Official Seed Analysts.

Ayerza, R. (1981). El Buffel Grass: utilidad y manejo de una promisoria gramínea.Buenos Aires, Argentina: Editorial Hemisferio Sur.

Bulat, H. (1961). Reduction processes in living tissue, formazan, tetrazolium salts and their importance as reduction-oxidation indicators in resting seed. En Proceedings of the International Seed Testing Association, 26 (686-696). Copenhagen, Denmark: International Seed Testing Association.

Copeland, T. G., Bruce, C. F. y Midyette, Jr. J.W. (1959). The unofficial application of tetrazolium test as an aid in checking germination claims. Proceedings of the Association of Official Seed Analyst (AOSA), 49 (134141). East Lansing, Michigan, U.S.A.

Delouche, J. C., Still, T. W, Raspet, M. y Lienhard, M. (1962). The tetrazolium test for seed viability (Technical Bulletin 51). Mississippi, EEUU: Mississipi State University.

Di Rienzo, J. A., Casanoves, F., Balzarini, M. G., Gonzalez, L, Tablada, M. y Robledo, C. W. InfoStat versión 2015. Grupo InfoStat, FCA, Universidad Nacional de Córdoba, Argentina. Recuperado de: http://www. infostat.com.ar

Ellis, R. H., Hong, T. D. y Roberts, E. H. (1985). Handbook of seed technology for gene banks (3), Vol. II Compendium of specific germination information and test recommendations. Rome, Italy: International Board for Plant Genetic Resources (IBPGR). Recuperado de: $\quad$ https://www.bioversityinternational.org/index. php?id $=244 \&$ tx news pi $1 \% 5$ Bnews $\% 5 D=147 \&$ tx news_pi $1 \% 5$ Bcontroller $\% 5 D=N e w s \& t x$ news_pi $1 \% 5 \mathrm{~B}$ action $\% 5 \mathrm{D}=$ detail \&cHash $=\mathrm{d} 6 \mathrm{db} 487481 \mathrm{db} 43 \mathrm{bfff} 3827$ $48 \mathrm{e} 6 \mathrm{f} 24 \mathrm{bc} 9$

Gosling, P. G. (2003). Viability testing. In: Smith R.D., Dickie J.B., Lenintong S.H., Pritchard H.W., Probert R.J. (Eds), Seed Conservation: Turning Science into Practice (445-481). Kew, U.K.: Royal Botanic Gardens.

International Seed Testing Association [ISTA] (2003). International Rules for Seed Testing. Working Sheets on Tetrazolium Testing. Zürich, Bassersdorf,Switzzerland: ISTA.
International Seed Testing Association [ISTA] (2012). International Rules for Seed Testing. Seed Science and Technology 31. Zürich, Switzzerland: ISTA.

Krzyzanowski, F. C., Vieira, R. D. y Franca Neto, J. B. (1999). Testes de Tetrazólio para Determinaçãodo Vigor de Sementes. Vigor de Sementes: Conceitos e Testes. Londrina, PR, Brasil: Abrates.

Moore, R. P. (1973). Tetrazolium staining for assessing seed quality. En: Heydecker, W. (Ed.) Seed Ecology(347-366). London, England: Butterworth.

Moore, R. P. (1985). Manual de ensayos al tetrazolio. Madrid, España: Instituto Nacional de Semillas y Plantas de Vivero.

Pérez, H. (2005). Características de las especies forrajeras adaptadas a las condiciones del Noroeste del país. (Documento no publicado). Hotel Holiday Inn, Córdoba, Argentina.

Peske, S. T., Rosenthal. M. D. y Madeiros Rota, G. R. (Eds.). (2003). Sementes: Fundamentos científicos e tecnológicos. Pelotas, Brasil: Rua.

Reartes, D. (2003). El futuro de la ganadería argentina. Recuperado de: http://www.produccion-animal. com.ar/informacion_tecnica/origenes_evolucion_y_ estadisticas_de_la_ganaderia/17-futuro_ganaderia argentina.pdf

Sharif-Zadeh, F. y Murdoch, A. J. (2000). The effects of different maturation conditions on seed dormancy and germination of Cenchrus ciliaris. Seed Science Research, 10 (4), 447-457. doi:10.1017/ $\underline{\mathrm{S} 0960258500000490}$

Smith, F. G. (1952). The mechanism of the tetrazolium reaction in corn embryos. Plant Physiology, 27(3), 445456.doi:10.1104/pp.27.3.445

Smith, F. G. y Throneberry, G. O. (1951). The tetrazolium test and seed viability. Proccedings of the Association of Official Seed Analysts, 41, 105-109. 